\title{
Behavioural Modelling for Adaptive Array Systems Incorporating High-Power Amplifier Nonlinearities
}

\author{
Tristan JD Goss ${ }^{1}$ and Peter H Aaen ${ }^{2}$ \\ ${ }^{1}$ Radar Sensing Team, Defence Science \& Technology Laboratory, Salisbury, United Kingdom, tjdgoss@dstl.gov.uk \\ ${ }^{2}$ Faculty of Electrical and Electronic Engineering, University of Surrey, Guildford, United Kingdom, p.aaen@surrey.ac.uk
}

\begin{abstract}
We develop a novel method for the behavioural modelling of adaptive array systems (AASs) incorporating highpower amplifier (HPA) nonlinearities. The model is motivated by the need to better define the spatial and spectral interference profile of AASs. We provide a simulated proof of concept as a precursor to experimental validation. In our demonstration, the model is identified using simulated near-field measurements made while the antenna's input channels are simultaneously undergoing a representative excitation. These measurements are used to identify a large number of memory polynomials (MPs) which characterise the transfer function from the antenna inputs to the near-field. We then use the array of MPs and a near-field to farfield transformation to obtain the far-field pattern of the antenna for arbitrary input. We show the impact of antenna element HPA nonlinearities on the far-field pattern, and our ability to predict them via the developed model.
\end{abstract}

Index Terms-adaptive antenna, smart antenna, behavioural model.

\section{INTRODUCTION}

An adaptive array system (AAS) is an antenna array in which each element has its own digital transmitter comprising a direct digital synthesiser (DDS) and a high-power amplifier (HPA), and its own digital receiver comprising a low-noise amplifier (LNA) and an analogue-to-digital-converter (ADC). AASs are likely to play an increasing role in future standards as an AAS can improve data rates and spectrum re-use over an antenna with a single fixed radiation pattern or a low number of switched patterns. In order to deploy any new radio frequency (RF) or microwave system, existing spatially and spectrally adjacent users must be assured that the interference that the new system will produce will not result in unexpected negative consequences for their systems.

The large number of antenna patterns that an AAS can generate makes it difficult to provide this assurance; an AAS's radiation pattern can be dependent on the number and location of served terminals, the propagation conditions and (especially for a wideband signal) the content of the transmission. The need to exhaustively simulate this behaviour in order to provide the necessary assurance can inhibit the deployment of AASs. This paper develops a method for creating a behavioural model of a given AAS. This behavioural model has a significantly reduced computational complexity compared to the physics-based electromagnetic (EM) models normally used, reducing the time taken to characterise the interference generated by the AAS. Our approach improves upon previous models by incorporating nonlinearities and memory effects of the HPAs with the AAS.

Both amplifier behavioural modelling and antenna source reconstruction have received significant attention and a number of summaries of the modelling of nonlinear power amplifiers with memory have been published by Isaksson, Pedro and others [1-5]. Recent developments in amplifier behavioural modelling are focussed on improving digital pre-distortion, while recent work on source reconstruction is focused on efficient wideband reconstruction [6]. These research areas are normally considered separated through the application of effective digital pre-distortion, which attempts to linearise the signals presented to the antenna elements. However, digital pre-distortion is not perfect, especially when its implementation is constrained by cost. Realistic AASs will exhibit residual nonlinearities due to design trade-offs, which will result in transmission into adjacent channels, the distortion of the symbols within the modulation scheme, the distortion of antenna patterns and the loss of power to adjacent frequencies. All of these effects contribute to power loss from the signal and make it harder to satisfy interference requirements. It is therefore prudent to incorporate HPA nonlinearities into an AAS behavioural model. Initial work has been completed which combines simpler nonlinear models with beamforming techniques [7].

In this paper, we present a behavioural model of the far-field radiation pattern of an AAS under arbitrary wideband signal excitation input, incorporating both amplifier nonlinearity and memory effects. Once validated, our model will enable faster characterisation of the spatial distribution of the co-channel and adjacent channel interference produced by the amplifier nonlinearities within an AAS than was previously possible, reducing the time taken to generate the evidence required for the deployment of an AAS to a new site. In this paper, we describe a proof of concept as a precursor to experimental validation of the approach.

\section{MOdEL DESCRIPTION}

The AAS behavioural model proposed here is a direct combination of the plane wave spectrum technique [8] for source reconstruction and the memory polynomial (MP) technique [9] for amplifier behavioural modelling. The MP technique was selected because it has been shown to more accurately model amplifiers with complex memory effects than the Wiener 
or Hammerstein models [3], because it requires significantly fewer parameters than a full Volterra series, and because it benefits from a linear model identification process. The plane wave spectrum technique for source reconstruction was selected because of its long pedigree, because it benefits from rapid computation via the fast fourier transform (FFT), and because it will be straightforward to apply to the planar nearfield measurement system used by our research group.

The model predicts each of three orthogonal electric field components $E_{x}, E_{y}$ and $E_{z}$ of the AAS near-field from the AAS inputs using different MPs, and then uses the plane wave spectrum technique to obtain the far-field from the near-field. The concept behind the model is shown in Figure 1. Each MP within the model corresponds to the contribution made to a single field component at a single near-field sample point by a single input port. As a result, for an AAS with $N_{i n}$ input ports, and a desired far-field resolution corresponding to $N_{N F}$ near-field sample points, the AAS behavioural model will contain $3 N_{i n} N_{N F}$ separate MP models.

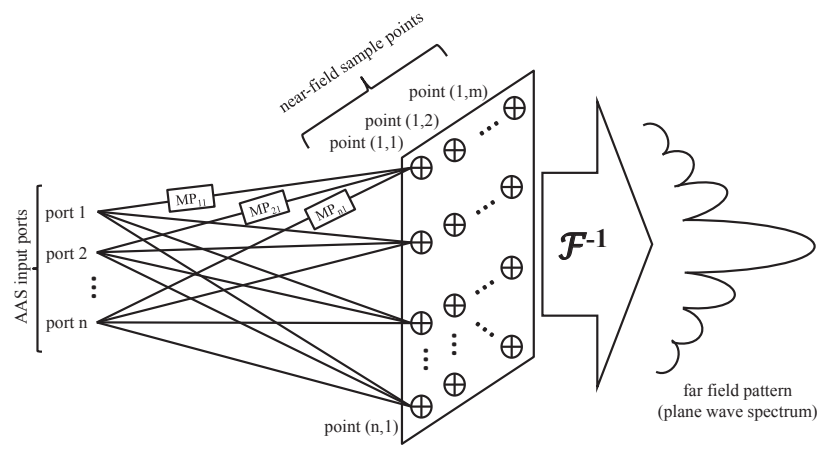

Fig. 1. The concept behind the proposed AAS behavioural model. To aid comprehension, only three MPs are shown, all of which refer to only one of three near-field components at a particular near-field sample point. In the proposed experimental system, the fields at each point will be measured by a near-field planar scanning system.

The MPs within the AAS behavioural model are not simply representations of the amplifier nonlinearity. They now embed the following behaviours:

- The effect of the amplifiers on the input signals, including their nonlinearity and memory effects.

- The radiation patterns of the antenna elements (which are sampled where necessary by each of the near-field measurement locations).

- Propagation delays from the antenna elements to the nearfield sample points. These are captured both in the phase of the MP coefficients and the memory delays within the MPs.

- Linear coupling between the antenna elements (assuming no power is coupled back into the amplifiers).

Our approach is valid for heterogeneous antenna elements, (including heterogeneity in both the element patterns and the amplifiers feeding the elements) and can easily be extended to other near-field scanning topologies.
Its key downside is a large number of coefficients, which arise as a result of the inability to separate out the antenna element positions and patterns from the amplifier behaviour when identifying the model. When the MP structure is the same for all of the MPs in the model, the number of coefficients $N_{\text {coef } f}$ within the model is given by:

$$
N_{\text {coeff }}=3(m+1) p N_{i n} N_{N F}
$$

where $m$ is the memory depth of the MPs and $2 p-1$ is the order of the (odd only) MPs. The factor 3 arises from the need to identify models for each orthogonal near-field component separately. In some cases the component normal to the scanning surface can be inferred from the other two, reducing the number of coefficients. Additional reductions in coefficient count can be obtained by reducing the near-field spatial sampling rate. Another disadvantage is that the model is incapable of representing AASs where the antenna elements are also nonlinear $[1,10]$. In order to predict the far-field for an arbitrary wideband excitation, the signal is first propagated through the MPs to obtain the near-field as a time series. This time series field is then Fourier transformed, and the plane wave spectrum found for each frequency individually.

\section{MODEL IDENTIFICATION}

The model is identified by simultaneously feeding representative signals into all of the input ports of the AAS, and recording a short segment of data at each of the near-field sample points. For example, if the AAS is to be used for 3rd generation partnership project (3GPP) long term evolution (LTE) signals, then LTE signals must be used to identify the model. The MPs related to each near-field sample point are identified separately, so it is not necessary for every sample point to record the result of the same segment of input data. The sample rate drives the accuracy of the model; increasing it allows for the unambiguous determination of higher order components and a finer resolution on the representation of the effects of memory, but also increases the computational difficulty of the identification. In order for the identification to function correctly, the input data presented to each AAS input port must be different so that the ports can be distinguished from each other in the near-field samples (which implies that the antenna under test (AUT)'s beam or beams must be steered in some direction other than broadside), as well as representative of all of inputs the AAS will receive during operation (i.e. including a sampling of all beam positions).

It is apparent from experimentation that as the difference between the input data presented to each channel increases, the number of samples required for successful identification reduces. It is likely that orthogonal signals will result in the fastest identification, provided the orthogonality condition does not impact upon the generality of the identified model. The identification proceeds similarly to the basic MP [11]. A linear identification system is formulated for each field component of each near-field sampling point in order to identify the model: 


$$
\vec{Y}_{i j}=\mathbf{X} \vec{A}_{i j}
$$

where $\vec{Y}_{i j}$ contains the measured samples of the $j$ th field component at the $i$ th near-field sample point, $\mathbf{X}$ is a regression matrix built using the input samples from all input ports, and $\vec{A}_{i j}$ contains the model coefficients to be identified, which correspond only to the $j$ th field component at the $i$ th nearfield sample point. The structure of the $i j$ th set of MPs, all of which are identified simultaneously, is determined by three coefficients: the memory depth $m$, the order $2 p-1$ and the number of input ports $N_{i n} . \vec{A}_{i j}$ therefore has dimensions $(m+1) p N_{i n} \times 1$. If $\vec{Y}$ has dimensions $y \times 1$, then $\mathbf{X}$ has dimensions $y \times(m+1) p N_{i n}$.

$\mathbf{X}$ is built by concatenating $m$ sub-matrices $\mathbf{G}(q)$ of dimensions $y \times p N_{i n}$, where each $\mathbf{G}(q)$ represents a particular delay value for all component MPs. For a set of MPs with $m=0$, only $\mathbf{G}(0)$ is present. The columns of $\mathbf{G}(q)$ refer to both different polynomial orders and different input ports while the rows of $\mathbf{G}(q)$ refer to different measured output samples. $\mathbf{G}(q)$ can be regarded as being constructed by concatenating together $N_{\text {in }}$ sub-matrices $\mathbf{H}(q, r)$ of dimensions $y \times p$ which each represent the $q$ th memory delay applied to the signal coming from the $r$ th input port. The elements of $\mathbf{X}$ are given by:

$$
\begin{aligned}
\mathbf{X} & =[\mathbf{G}(0) \cdots \mathbf{G}(q) \cdots \mathbf{G}(m)] \\
\mathbf{G}(q) & =\left[\mathbf{H}(q, 0) \cdots \mathbf{H}(q, r) \cdots \mathbf{H}\left(q, N_{i n}\right)\right] \\
\mathbf{H}(q, r)_{k l} & =u_{r}(k-q)\left|u_{r}(k-q)\right|^{2(l-1)}
\end{aligned}
$$

where the indexes $k$ and $l$ into $\mathbf{H}(q, r)$ are used to deconflict with the unrelated use of $i$ and $j$ in (2) and $u_{r}(k)$ is the $k$ th sample of input data at input port $r$. (2) is then solved using the least squares method as with the basic MP:

$$
\vec{A}_{i j}=\left(\mathbf{X}^{H} \mathbf{X}\right)^{-1} \mathbf{X}^{H} \vec{Y}_{i j}
$$

where $\mathbf{X}^{H}$ denotes the Hermitian transpose of $\mathbf{X}$. The correct value for these coefficients is determined using a performancedriven sweep method with the root mean squared error (RMSE) used as the performance metric. The identification method is then:

1) Select a maximum RMSE threshold based on the desired model accuracy.

2) Select a maximum model complexity $c_{\max }$, and then select all combinations of $p$ and $m$ such that $1 \leq p, p \in$ $\mathbb{Z}, 0 \leq m, m \in \mathbb{Z}$ and $(m+1) p \leq c_{\max }$ as candidate models.

3) Pass a representative signal through the amplifier large enough to reach the amplifier's nonlinear region.

4) Divide the signal and the amplifier output into training and test portions.

5) While the RMSE is above the threshold and untested models remain:

a) Select the next least complex model. b) Solve (6) for the selected model using the training portion of the signal and the corresponding amplifier output.

c) Attempt to predict the amplifier output from the training portion of the signal using the identified MP, and measure the RMSE of the prediction.

d) If the RMSE remains above the threshold, select the next least complex model and try again.

6) If all combinations of $m$ and $p$ have been tried, select a larger $c_{\max }$ and try again from step 2 .

7) Use the identified MP to predict the amplifier output for the test portion of the signal, and measure the RMSE of the prediction.

8) If the RMSE is close to the threshold defined in step 1 (e.g. within $1 \mathrm{~dB}$ ) when applied to the test data, the process is complete.

9) If the RMSE is more than $1 \mathrm{~dB}$ above the threshold defined in step 1 , the model is over-fitted and the process must be repeated from step 3 with a longer representative signal.

To ease computation and to cut down on the prodigious number of coefficients, the method should be applied separately to each near-field element, rather than in a way that measures its completeness using RMSE figures for the entire near-field at once.

\section{Application And Demonstration}

\section{A. Scenario}

We provide an example in which an AAS composed of 8 Hertzian dipoles in a linear array is used to transmit two LTE signals concurrently at different frequencies (separated by $30 \mathrm{MHz}$ ) in different directions using delay-and-sum digital beamforming (DBF). This example was produced using a bespoke simulation environment built using MATLAB $\AA$ Simulink ${ }^{\circledR}$, including the digital signal processing (DSP) System Toolbox ${ }^{\mathrm{TM}}$ and the RF Blockset ${ }^{\mathrm{TM}}$. The simulated nonlinear HPAs are based on datasheets [12] describing the MACOM $^{\mathrm{TM}}$ MAAP-010169 pseudomorphic high electron mobility transistor (pHEMT) implemented within the behavioural amplifier model within the Simulink RF Blockset. Where "linear" amplifiers are described, this refers to a simpler, linearised model of the MAAP-010169. Auxiliary MATLAB ${ }^{\circledR}$ scripts were used in order to simulate the electric field in both cartesian (for the near-field) and spherical (for the far-field) coordinates.

Plots of the magnitude of the $\theta$ component of the electric field $\left|\vec{E}_{\theta}\right|$ versus frequency and azimuth for this example AAS using both linear and nonlinear amplifiers are shown in Figures 2 and 3. Two effects of the amplifier nonlinearities on the beam pattern are apparent: the radiated power at most combinations of azimuths and frequencies is increased, and the structure and position of the adjacent channel sidelobes has changed. These effects are examples of the complex behaviour that we seek to predict with the AAS behavioural model. 


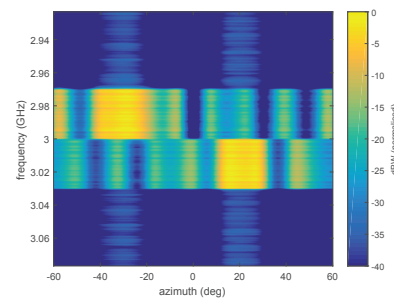

Fig. 2. Frequency versus azimuth plot of $\left|\vec{E}_{\theta}\right|$ at $0^{\circ}$ elevation of the example AAS using linear amplifiers.

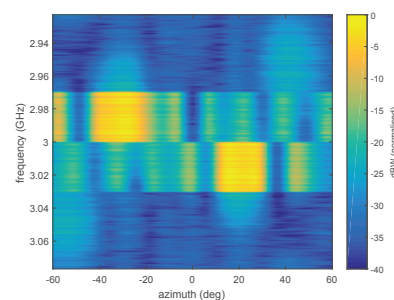

Fig. 3. Frequency versus azimuth plot of $\left|\vec{E}_{\theta}\right| 0^{\circ}$ elevation of the example AAS using nonlinear amplifiers.

\section{B. Identification}

An AAS behavioural model was identified across $21 \times$ 21 near-field sample points separated by $0.5 \lambda$. In order to represent every possible combination of beam positions, azimuth beam positions spanning $120^{\circ}$ sampled at $5^{\circ}$ intervals (in order to keep the total computation low) were defined, and then all combinations of two of these beam positions were defined for a total of 625 beam position combinations. For each combination, two short LTE test signals comprising approximately 100 symbols were transmitted at two different frequencies aimed at the respective beam positions. The total duration of all test signals was $2.08 \mathrm{~ms}$, which through experimentation was shown to be approximately the minimum necessary to prevent model over-fitting (which was defined as exceeding $2 \mathrm{~dB}$ over the RMSE threshold on the test portion of the data).

The RMSE threshold was set independently for each field component of each near-field sample point, at $10 \mathrm{~dB}$ below the mean amplitude of the field at that point. The full identification took 2 hours and 38 minutes using a Toshiba Tecra R9501EM Laptop, although it should be noted that this time also includes the computation of the simulated near-field samples. The identification of each near-field sample is unrelated to the identification of the others, so the process can be readily accelerated via parallel computation. The identified model was $0.88 \mathrm{MB}$ in size and featured a total of 54,872 coefficients.

\section{Performance}

The RMSE in the cartesian $z$ component of the electric field $\left|E_{z}\right|$ when the AAS behavioural model is used is 0.89 $\mathrm{dBm}$, while the RMSE displayed by a model ignoring amplifier nonlinearity is $10.89 \mathrm{dBm}$ (note that both figures are measured across azimuth and frequency as per Figures 4 and 5). The AAS behavioural model predicted far-field for a particular combination of beam positions is shown in Figure 4. The directly simulated far-field is shown in Figure 5. The close agreement of these two images demonstrates the correct functioning of the AAS behavioural model. Figure 6 shows the distribution of the errors in the predicted far-field. This demonstrates the added value of the AAS behavioural model over an approach which does not reflect amplifier nonlinearity. While these specific numbers were obtained for a particular set of beam positions, the RMSE performance reported during the model identification indicates that similar performance can be expected for other beam positions.

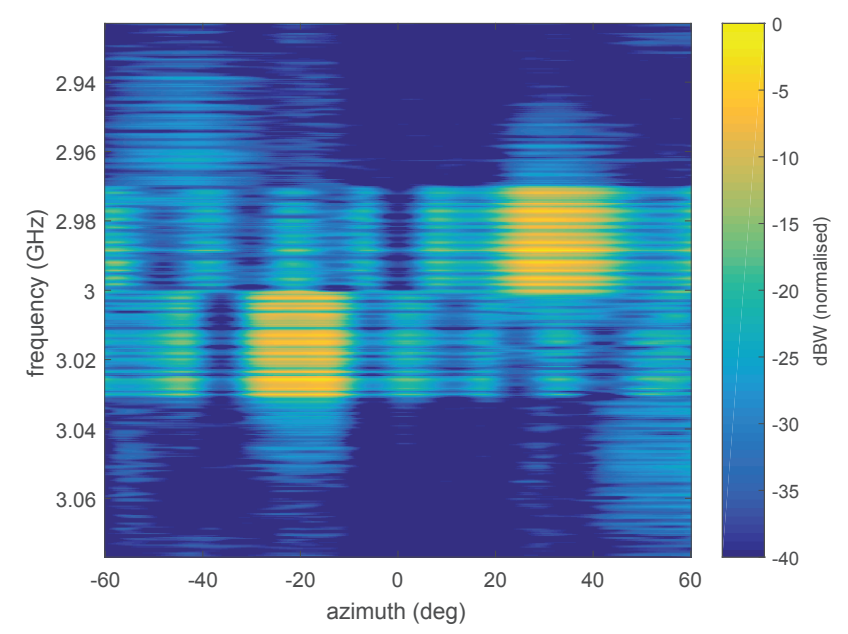

Fig. 4. Prediction of a frequency versus azimuth plot of $\left|\vec{E}_{\theta}\right|$ at $0^{\circ}$ elevation for the LTE signal using the AAS behavioural model.

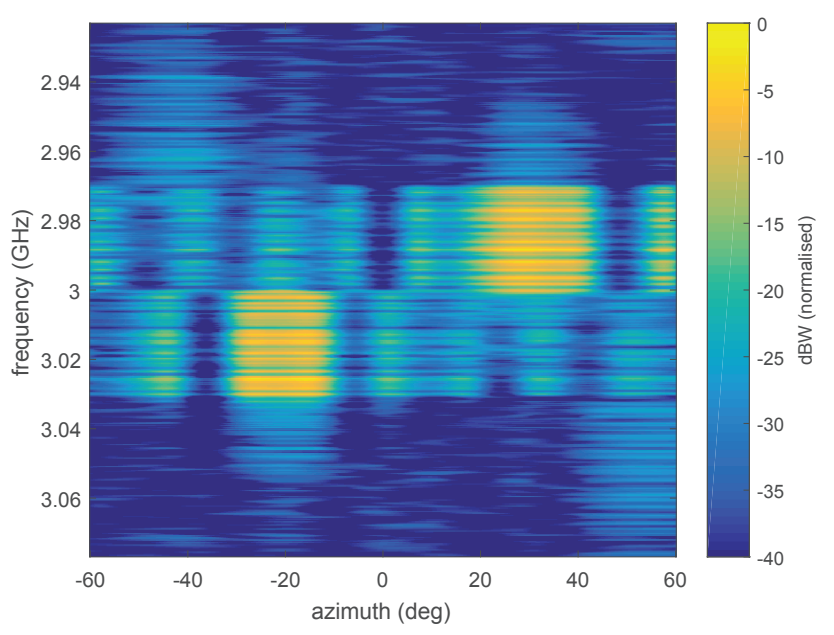

Fig. 5. Frequency versus azimuth plot of $\left|\vec{E}_{\theta}\right|$ at $0^{\circ}$ elevation produced by the example AAS using nonlinear amplifiers.

The accuracy of the prediction is not the only relevant figure of merit: the time taken to make the prediction is also important. On a Toshiba Tecra R950-1EM laptop, the AAS behavioural model took approximately 3.53 seconds to predict the near-field for 512 input samples (spanning $3.34 \mu s$ ), and a further 1.5 seconds to transform the near-field predictions to the far-field.

\section{CONCLUSION}

This paper presents a novel AAS behavioural model that can be identified without any knowledge of the internal workings of an AUT and then used to rapidly predict the far-field radiation produced by the AUT for an arbitrary input signal. The intended use for this model is the accurate prediction of 


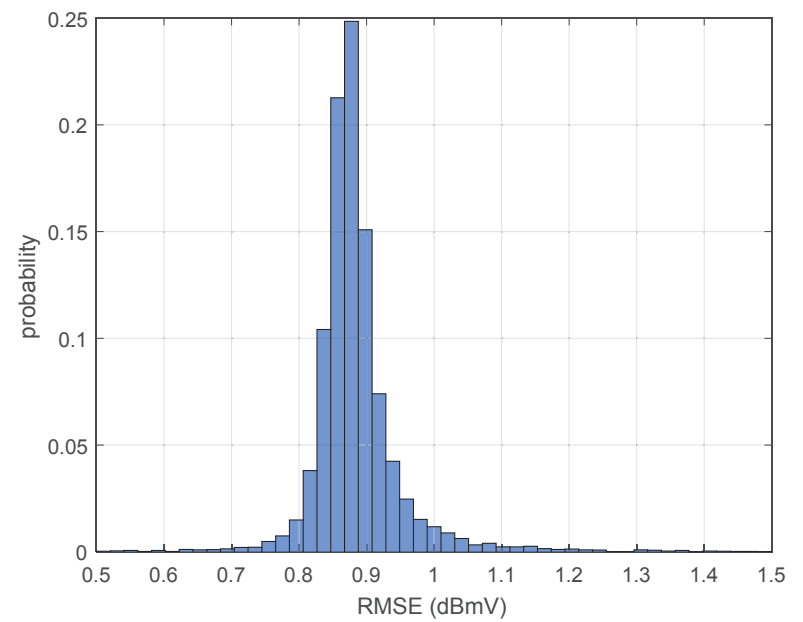

Fig. 6. Histogram of the error in the predictions of $\left|\vec{E}_{\theta}\right|$ made by the AAS behavioural model.

the electromagnetic interference (EMI) produced by the AUT for provision to spatially and spectrally adjacent RF system users in order to ease their granting of permission for the deployment of the AUT.

This paper has presented a behavioural model, demonstrated its correct function in simulation and made comments on its performance. Its chief limitations are its large number of coefficients and its relatively slow and data-heavy identification process. Many of these limitations can be mitigated through exploitation of the highly parallel architecture of the model. Future research will focus on combining this model with farfield reconstruction techniques which require fewer near-field samples, and attempting to make use of different amplifier modelling approaches in place of the MP.

\section{ACKNOWLEDGMENTS}

The authors would like to acknowledge Dr Tim Brown of the University of Surrey, Faculty of Electrical and Electronic Engineering, for his leadership of the MSc by Short Course programme which facilitated this work, Dr Aaron Jones of the US Air Force Research Laboratory for his review of early drafts of this paper, Mr Anthony Barfoot of the the Dstl Contested Electromagnetic Environments research programme for funding the latter portions of the work, and the Engineering and Physical Sciences Research Council (EPSRC) for a Strategic Equipment Grant (EP/L02263X/1), which supported the development of this work.

\section{LICENSING}

This material is licensed under the terms of the Open Government Licence except where otherwise stated. To view this licence, visit http://www.nationalarchives.gov.uk/doc/opengovernmentlicence/version/3 or write to the Information Policy Team, The National Archives, Kew, London TW9 4DU, or email: psi@nationalarchives.gsi.gov.uk.

\section{REFERENCES}

[1] P. Roblin, D. Root, J. Verspecht, Y. Ko, and J. P. Teyssier, "New trends for the nonlinear measurement and modeling of high-power RF transistors and amplifiers with memory effects," IEEE Trans. Microw. Theory Techn., vol. 60, no. 6, pp. 1964-1977, June 2012.

[2] P. Landin, M. Isaksson, and P. Handel, "Comparison of evaluation criteria for power amplifier behavioural modeling," in IEEE MTT-S Int. Microw. Sym., Atlanta, USA, June 2008.

[3] M. Isaksson, D. Wisell, and D. Ronnow, "A comparative analysis of behavioural models for RF power amplifiers," IEEE Trans. Microw. Theory Techn., vol. 54, no. 1, pp. 348-359, January 2006.

[4] A. S. Tehrani, H. Cao, S. Afsardoost, T. Eriksson, M. Isaksson, and C. Fager, "A comparaive analysis of the complexity/accuracy tradeoff in power amplifier behavioural models," IEEE Trans. Microw. Theory Techn., vol. 58, pp. 1510-1520, 2010.

[5] J. C. Pedro and S. A. Maas, "A comparative overview of microwave and wireless power-amplifier behavioral modeling approaches," IEEE Trans. Microw. Theory Techn., vol. 53, no. 4, pp. 1150-1163, April 2005.

[6] P. Li, Y. Li, L. J. Jiang, and J. Hu, "A wide-band equivalent source reconstruction method exploiting the StoerBulirsch algorithm with the adaptive frequency sampling," IEEE Trans. Antennas Propag., vol. 61, no. 10, pp. 5338-5343, October 2013.

[7] E. C. Real and D. P. Charette, "Non-linear amplifier effects in transmit beamforming arrays," in IEEE Int. Conf. on Acoustics, Speech, and Sig. Proc., vol. 5, May 1995, pp. 3635-3638.

[8] E. C. Burdette, "Plane wave spectrum analysis of nearzone field using wavenumber and band-pass filtering," Ph.D. dissertation, Georgia Institute of Technology, May 1967.

[9] H. Ku and J. S. Kenney, "Behavioral modeling of nonlinear RF power amplifiers considering memory effects," IEEE Trans. Microw. Theory Techn., vol. 51, pp. 24952504, Dec 2003.

[10] B. K. Meadows, T. H. Heath, J. D. Neff, E. A.Brown, D. W. Fogliatti, M. Gabbay, V. In, P. Hasler, S. P. Deweerth, and W. L. Ditto, "Nonlinear antenna technology," in Proc. of the IEEE, vol. 90, May 2002, pp. 882-897.

[11] A. E. Abdelrahman, O. Hammi, A. K. Kwan, A. Zerguine, and F. M. Ghannouchi, "A novel weighted memory polynomial for behavioral modeling and digital predistortion of nonlinear wireless transmitters," IEEE Trans. Ind. Electron., vol. 63, no. 3, pp. 1745-1753, March 2016.

[12] MAAP-010169 10W Power Amplifier, web resource, MACOM, August 2017. [Online]. Available: https://www. macom.com/products/product-detail/MAAP-010169 